\title{
Feasibility and safety of minilaparoscopy-guided spleen biopsy
}

\author{
Yunqiang Cai $\cdot$ Xubao Liu
}

Received: 29 December 2012/ Accepted: 21 January 2013/Published online: 12 March 2013

(c) Springer Science+Business Media New York 2013

To the Editor,

We read with interest the article by Werner et al. [1]. We appreciate their effort to clarify the usefulness of this technique in practice of establishing the diagnosis of splenic abnormalities. However, we have several concerns about this technique.

In the setting of cystic tumors or some inflammatory lesions such as tuberculosis, it is difficult to guarantee that cells will not be disseminated in the peritoneal cavity in the process of spleen biopsy. There was no peritoneal disseminations reported in the series. I hope the authors can share their experience in preventing peritoneal dissemination.

During the process of minilaparoscopy-guided spleen biopsy, we wonder how the authors located the lesions that were in the parenchyma of the spleen.

We are also concerned with the indications of this procedure. In our opinion, it is not suitable for all patients with focal splenic lesions detected in prior imaging studies to undergo minilaparoscopy-guided spleen biopsy. For many lesions, such as hemangioma and tuberculoma, mere diagnosis of these abnormalities is insufficient. Treatment, such as splenectomy or partial splenectomy, is required. During the last decade, which has seen a strong emphasis on the preservation of the immunologic function of the spleen, there has been an increased interest in performing a partial splenectomy for a number of indications, including nonparasitic cysts, benign tumors, and lymphoma staging
$[2,3]$. In the setting of these lesions, we prefer splenectomy or partial splenectomy to spleen biopsy for the reason that splenectomy or partial splenectomy not obtain more tissue for histopathologic examination while simultaneously removing the lesions.

We agree with Werner et al. that minilaparoscopyguided spleen biopsy is an effective and safe procedure to establish a definitive diagnosis for many patients. However, we think that it is not suitable for all patients with focal splenic lesions found via prior imaging studies. The selection criteria for this technique should be better defined.

Disclosures Drs. Yunqiang Cai and Xubao Liu have no conflicts of interest or financial ties to disclose.

\section{References}

1. Werner T, Koch J, Frenzel C, Lohse AW, Denzer UW (2012) Effectiveness and safety of minilaparoscopy-guided spleen biopsy: a retrospective series of 57 cases. Surg Endosc 26:2416-2422

2. Dutta S, Price VE, Blanchette V, Langer JC (2006) A laparoscopic approach to partial splenectomy for children with hereditary spherocytosis. Surg Endosc 20:1719-1724

3. Hong TH, Lee SK, You YK, Kim JG (2010) Single-port laparoscopic partial splenectomy: a case report. Surg Laparosc Endosc Percutan Tech 20:e164-e166
Y. Cai $\cdot$ X. Liu $(\bowtie)$

Department of Hepatopancreatobiliary Surgery, West China

Hospital, Sichuan University, No 37, Guo Xue Xiang,

Chengdu 610041, Sichuan, China

e-mail: shoubulele@gmail.com 